Stellenweise wurde auch fette Lehmerde zugesetzt.

Die Brotersatzstoffe und namentlich auch die eigentlichen Hungerbrote enthielten nach der chemischen Untersuchung im Vergleiche mit gewöhnlichem Roggen- und Weizenbrote bedeutende Mengen Proteinstoffe, theilweise auch Fett, daneben aber auch reichliche Mengen Rohfaser und Mineralstoffe. So namentlich die aus Eichelmehl und unter Verwendung von Sonnenblumensamenrückständen, Unkrautsamen, Stroh u. s. w. hergestellten Brote. Der Gehalt an Rohfaser belief sich in diesen Brotersatzstoffen in der Trockensubstanz bis auf $20 \%$ und mehr, der Gehalt an Mineralstoffen auf $10-12 \%$, in einem mit fetter Lehmerde bereiteten Brote auf $64 \%$.

Die vorgezeigten Brotproben, namentlich die eigentlichen Hungerbrote, hatten in Farbe und Aussehen eine von unseren gewöhnlichen Brotsorten erheblich abweichende Beschaffenheit. So hatte z. B. das unter Verwendung von Schilfwurzeln hergestellte Brot in Farbe und Struktur grösste Aehnlichkeit mit Filzplatten, wie solche als Bierglasuntersätze Verwendung finden. Das aus Sonnenblumensamen-Rückständen bereitete Brot hatte ein tiefschwarzes kohliges Aussehen. Letztere Brotsorte soll auch einen derartig widerwärtigen Geschmack besitzen, dass selbst für Hungernde der Genuss eine grosse Ueberwindung bedeutet.

Die mit den verschiedenen Brotersatzstoffen - ausschliesslich der eigentlichen Hungerbrote - angestellten Ausnutzuugsversuche haben, auf Trockensubstanz berechnet, für dieselben eine Ausnutzung ergeben, welche selten schlechter als wie bei Schwarzbrot (etwa $70 \%$ ) war.

Bei den eigentlichen Hungerbroten belief sich die Ausnutzung auf $40-50 \%$. $\mathrm{Zu}$ bemerken ist noch, dass bei diesen Ausnutzungsversuchen der Körper der Versuchspersonen in keinem Falle im Stickstoffgleichgewichte gehalten werden konnte.

Vergl. hierzu auch die Mittheilung von A. Mauricio in dieser Zeitschrift 1901, 4, 1017-1019 und die Nachschrift zu derselben von A. Bömer. K. Lendrich.

\title{
Der Stand der Abwässerreinigungsfrage auf Grund praktischer Versuche in Württemberg.
}

Von Medicinalrath Dr. Scheurlen in Stuttgart.

Vortragender berichtet über zwei an württembergischen Anstalten nach dem biologischen Verfahren eingerichtetè Abwässer-Reinigungsanlagen, nämlich in der Lungenheilanstalt Wilhelmsheim und in der Irrenanstalt Winnenthal. Die Kläranlagen sind dort nach demselben Princip angelegt wie die Versuchskläranlage des Hygienischen Instituts zu Hamburg und bestehen aus einem Vorraum, sogen. Faulkammer, zur Aufnahme und Ansammlung der Abwässer und zwei hintereinander geschalteten Filtern. Als Filtermaterial dienen Koks und Schlacke von $3-5 \mathrm{~mm}$ Korngrösse. Die Abwässer bestehen aūs den Abort- und Küchenabgängen und Regenwasser.

Nach Vortragendem arbeiten die Anlagen bereits seit über einem Jabre in sehr zufriedenstellender Weise. Bereits nach dem Durchlaufen des ersten Filters ist eine Abnahme der organischen Substanz um $50 \%$ zu verzeichnen und die vom zweiten Filter kommenden Abwässer sind, wie auch die herumgezeigten Proben heweisen, vollkommen fäulnissfrei.

An dem Vortrag schloss sich eine lebhafte Disskusion an.

K. Lendrich. 